\title{
A Notification System Model for Bioinformatics Community of Practice
}

\author{
Rusli Abdullah \\ Faculty of Computer Science and Information Technology, \\ University Putra Malaysia, 43400 UPM Serdang, Selangor, Malaysia \\ E-mail: rusli@fsktm.upm.edu.my \\ Norhashimi Mohd Nor \\ Information Management Division, Ministry of Education Malaysia \\ Level 4, Block E11, Kompleks E, Precint1, 62604 Putrajaya \\ E-mail: norhashimimohdnor@moe.gov.my \\ Amir Mohamed Talib \\ Faculty of Computer Science \& IT, University Putra Malaysia \\ 43400 UPM, Serdang, Selangor, Malaysia \\ E-mail: ganawa53@yahoo.com
}

\begin{abstract}
Bioinformatics can be considered as a new field of study and it promises a vast exploration area (Carzaniga., Rosenblum., Wolf ., 2001). In order to expedite the maturity in this area, a proper and supportive portal where all researchers could gather and cooperate in conducting their research need to be established. One of the features in a portal that can assist Bioinformatics researchers in performing their work is the ability of the portal to notify. Notification system is a combination of software and hardware to provide a method of distributing message(s) to a set of recipients. The notification messages could assist the recipients in many ways, from time saving and cost saving till life saving. Notification system can be developed with numerous functions depending on the needs and one of the most beneficial functions in research area is notification on the next most relevant knowledge to be read. This type of notification could lessen the researchers' time in finding the correct thus enhancing their research efficiency. Another type of notification that could assist researchers is the events reminder. Busy and hectic researchers could forget their pack schedule and put total focus on their research while the reminder prompts them when it is time through Windows' pop up, email and SMS as the means of delivering the messages. Whilst the knowledge management system (KMS) provides a sturdy basic for the Bioinformatics portal as a whole, the agent technology support the operation of the notification system. Agent technology offers great capabilities in ensuring the recipients is notified accordingly through its autonomous, learning and cooperative characteristics. The objective of this project is to build a notification system for Bioinformatics community of practice $(\mathrm{CoP})$. Researchers in this community could utilize this system to make their research process more efficient.
\end{abstract}

Keywords: Notification, Notification system, Reminder, Agent technology, Knowledge management system and bioinformatic

\section{Introduction}

"Knowledge is power" is a famous quote when it comes to competition. Despite the field or the player, knowledge is the most powerful tool in securing victory. Since the creation of internet, knowledge is available anytime, anywhere on our finger tips it is growing larger and larger. This information overload is made "worse" by "data smog", the proliferation of low quality information because of easy publication says (Heylighen, 2002).

Abundant of knowledge does not always mean good to us if we do not have proper skills or tools to filter and notify only the needed knowledge. Thus in this paper one of the focus is to filter and notify only the needed knowledge from the ample knowledge available. In this study, the authors will focus on Bioinformatics community and develop a system that could help users in the Bioinformatics community to get notification only 
on related and needed knowledge. The Bioinformatics community means a group of people that share same interest in Bioinformatics, and they could be academician, researcher, sponsor, businessman and students.

(Heylighen, 2002) stated that, we are forced to consider more information than we can effectively cater. It is true because our world today is full of information and we have so little time to dig to all the information to get what we really want. Therefore it is important for us to know how to select the most important information so that we can get the best result with the least time.

Besides difficulty in selecting the right information, we are also burden with remembering our daily tight schedule. Our dairy or planner is a helpful media for keeping our schedule but it doesn't notify us about coming activities. Microsoft Outlook is another option for us to be notified but windows pop up and email message are not enough since we are not always in front of our computer.

Due to the lack of notification mechanism in bioinformatics community, the authors would like to propose a notification system that able to notify users in the bioinformatics community. The notification system in bioinformatics community will be developed based on the model that will be proposed in the later section 2 .

\section{Literature Review}

\subsection{Notification in Bioinformatics}

Notification comes from the word notify means telling, informing, alerting etc. When we are discussing about notification in general, it involves in our daily lives. A microwave buzz means the food that we cook is ready, a door bell rings means there are somebody at the door, car reverse sensor buzz means the car rear is too close to an object and etc.

Notification can help us in remembering something that we have to do, for example reminding us about our coming activities in our daily schedule. Not everybody can afford to hire a PA to remind us about our next activity, while dairy is a good schedule keeper but not a reminder.

In IT (Information Technology), notification can exist in a simple form for example an e-mail noting when a page has been inserted into a website is adequate for such a straightforward task. On the other hand, notifying targets when a building is on fire or nuclear leakage at nuclear power reactor would require real-time system. The bottom line of notification is to inform the recipients about something and they should react accordingly to the message.

Based on (Carzaniga., Rosenblum., Wolf., 2001) an event notification service implements two key activities, notification selection and notification delivery. The event or in this project context the articles need to be advertise and publish by the owner to the server. Then the interested party or user of the system subscribes to any categories of article available and get notify when articles in their selected categories are available. In Carzaniga et el research, because of some issues the system is modelled as a distributed system. The architecture comprises of a few interconnected servers to cater the complexity of the system's content as shown in Figure 1. However in our project we are going to develop single server architecture as it fits our requirements (Note 1).

There are a few Bioinformatics portals available on the internet such as Wageningen Bioinformatics (Wageningen Bioinformatics Webportal, Last Modified July 22, 2009 by JL, http://www.bioinformatics.nl/ ; CGB Bioinformatics Portal http://bioportal.cgb.indiana.edu/ ; BioPlanet .The Bioinformatics Homepage, http://www.bioplanet.com/ ; Bioinformatics Organization at http://www.bioinformatics.org/) and a lot more. They host generously amount of knowledge in Bioinformatics and very suitable for researchers that hunger for Bioinformatics resources. Generally most of the portals are equipped with search function that allows users to find articles that they wish for easily. They organize the articles or posts by categories so that it is easy for users to browse through.

However, none of the portals is equipped with notification facility in particular notification on related articles and schedule reminder. As a result we believe that there is room for the author to explore and develop a notification system for Bioinfomatics CoP.

"Bioinformatics is the application of computer technology to the management of biological information" (http://www.bioplanet.com/whatis.html). It is related to techniques and concepts from information, statistics, mathematics, chemistry, biochemistry, physics, and linguistics. Bioinformatics is applied in the creation and maintenance of a database to store biological information at the beginning of the "genomic revolution", such as nucleotide and amino acid sequences. 
In bioinformatics, IT are utilized to gather, store, analyze and integrate biological and genetic information which can then be applied to gene-based drug discovery and development (Bioplanet, http://www.bioplanet.com/whatis.html).

(Jacques C., (2004)) stated that he believes that the ultimate goal of bioinformatics is to develop in silico models that will complement in vitro and in vivo biological experiments. Where silico models are models that uses technology and algorithm to predict the result of a research rather than a thorough animal and clinical studies (Morrow K. J, 2008).

Notification has a strong role in bioinformatics. One of the obvious role is to notify the community on new available article or knowledge. To make this happen a portal need to be developed for the bioinformatics community to interact and work among themselves. In the portal, the bioinformatics community can get knowledge and contribute knowledge on bioinformatics. Besides that, they can also discuss and exchange ideas and vision on their research among themselves. One of the most precious potential of notification in the bioinformatics portal is to correlate among knowledge.

As we know bioinformatics is tool to analyze and integrate biological and genetic information to create new drugs and possibly food. Through notification in bioinformatics, researchers are assisted with tools that can propose ideas on their research. The notification ability in bioinformatics can correlate content of knowledge to another potential knowledge.

\subsection{Knowledge Management System in Bioinformatics}

As mentioned in earlier subtopic, bioinformatics is related with techniques and concepts from information, statistics, mathematics, chemistry, biochemistry, physics, and linguistics. It can be defined as knowledge of biological data from computer analysis and obviously it needs a proper system that can manage the massive knowledge. Besides the data generated by computer analysis, the needs of KMS is urged by the necessity of the researchers in the bioinformatics community to communicate and exchange ideas among themselves. The strategy and ultimate target of implementing KMS in bioinformatics community is to become a "Learning Organization" (InfoRouter- Knowledge Management, http://www.inforouter.com/).

In implementing KMS in bioinformatics institution, (Abdulla R., 2008) emphasize on three vital components; humans, technologies and bioinformatics content development to serve the Community of Practice $(\mathrm{CoP})$ of bioinformatics. They also proposed the model of bioinformatics KMS framework as shown in Figure 2 (Note 1).

\subsection{Agent Technology}

(Russell S., \& Norvig P. A., 1995) defined agent as "An agent is anything that can be viewed as perceiving its environment through sensors and acting upon that environment through effectors", as illustrated in Figure 3 (Note 1). (Russell S., \& Norvig P. A., 1995) stated that a human agent has eyes, ears, and other organs for sensors, and hands, legs, mouth, and other body parts for effectors. While a robotic agent substitute cameras and infrared range finders for the sensors and various motors for the effectors. In software, precepts and actions of agents are encoded in bit strings.

During their studies, (Rodríguez O. M., Vizcaino A., 2004) realized that there are several reasons why agents are good technical alternatives for KM software. According to Rodriquez, agents are proactive that is able to act automatically when it is necessary, while in some school of taught they called it autonomous. (Nwana H. S., 1996) stated that "Autonomy refers to the principle that agents can operate on their own without the need for human guidance". Besides that, (Abdulla R., 2008) stated that the second attribute that should exist in agents is their ability to cooperate and negotiate. The last attribute is the agent ability to learn by themselves in improving their performance over time.

Agent Topology in Figure 4 (Note 1) shows how the three (3) attributes correlate with each other to form four (4) different kinds of agents, collaborative agent, collaborative learning agent, interface agent and the ultimate smart agent that comprises all attributes.

\section{Methodology}

The methodology process starts from the ground work or information seeking activities, analyzing the findings gathered, designing the system based on analysis results, developing or coding the system adhere to the design, testing the completed system and lastly deploying the system into real environment. The long and continuous process is called the system development life cycle (SDLC).

The requirement seeking and gathering process is intensified and focuses specifically on the software. We played the role of an "analyst" must understand the information domain for the software, as well as required function, 
behaviour, performance and interface. At this stage, the "analyst" focused on "what" not "how". For example, what data does the system produce and consume, what functions the system must perform, what behaviours does the system exhibit, what interfaces are defined and what constraints apply.

\subsection{Conceptual Design}

During this phase, upon evaluating current problems, desired information (input and output) and outcome of the project, we began to see one or more solutions. From the preliminary investigation result and since the target group of the system is the Bioinformatics Community of Practice that need to do their research on bioinformatics, immediately the author had in mind, they have vast knowledge on Bioinformatics and they need even more knowledge. Other than that, they are the type of people that have lots of work and ideas in mind with little time in hands and therefore the ultimate goal in building this system is to assist them in conducting their research by lessen their tasks and chores.

Some of the essential requirements in assisting them are:

a) Lessen their time in searching for the correct knowledge or articles for their research.

b) Allow them to forget about their daily schedule and let them focus on their research.

c) Enhance their communication and collaboration among the community to promote research in Bioinformatics.

In this section the authors will elaborate on system development model that been used, system design and development process. System design will be from the software requirements, the system requirements and system architecture. These activities could facilitate the author in assembling and documenting the information in an organized and meaningful manner. Afterwards, the system implementation will be performed based on the system design.

Architectural design represents the structure of the program components that are required to build a computer-based system. It provides the big picture of a system. Architecture at the most simplistic level, it is analogous to the architecture of a building (Pressman R.S., 2001). It is the manner in which various components of the building are integrated to form a cohesive whole. From the problem statement that have been mentioned in the last sentence at the introduction section above and the literature review, we came out with architecture to solve the problem mentioned. Figure 5 describes simply the components of the system and their interactions (Note 1).

This section will explained the diagrams used in this system. Figure 6 (Note 1) shows the use case diagram of the system. From the use case, it will show the actors involved in the system. In this system, the actors involved are User, Administrator and Notification Agent. Besides that, through the use case diagram the roles of each actor can be depicted clearly and it also shows which system function will be performed by which actor.

Activity diagrams describe the behaviour of a system in terms of activities, says (Bruegge B. \& Dutoit A. H., 2004). They are loosely defined diagram technique for showing workflows of stepwise activities and actions, with support for choice, iteration and concurrency. The activity diagram below (Figure 7) shows the posting of new article activities flow (Note 1$)$.

\subsection{Physical Design}

User interface module is the front end of this system. The main purpose of this module is to provide the interface to the user for them to communicate with the system. From the interface, the user can key-in or click their request and then send the request to the application to be processed. Besides that, the interface module also will display the output from the system to the user.

There are nine (9) main components in Notification System for CoP interface design. They are:
a) Login
b) Main page
c) Articles Categories
d) Full Article reading and comment
e) Article Submission
f) Article Amendment
g) Community Events
h) My Calendar (Schedule) 
i) Personal Details

In the application module, the system function will reside here. The user can find solution available in the system. Besides that, they user are able to post new article, read and comment existing articles, edit and delete their own. Some of the tools available in the system are:

i. Post new article

ii. Read existing articles

iii. Comment existing articles

iv. Edit their own articles

v. Delete their own articles

vi. Search articles

vii. Create reminder

viii. Customize reminder

\section{Evaluation}

A group of ten (10) System Analyst and ten (10) Master's students were asked to evaluate the system and give their opinion about the system.

The author has design a simple questionnaire with the purpose of surveying the functionality, usability (ease of use) and efficiency of the system. The questionnaire is divided into 3 sections:

- $\quad$ Section A: General (user information)

- Section B: System (functionality, usability and efficiency)

- Section C: User suggestion

Before the evaluation session, participants were given short briefing on Notification System for Bioinformatics CoP. Besides that they were also given a list of functions that they should evaluate and questionnaire form. After that the participants were left alone to explore the system by themselves and fill in the questionnaire form in 15 minutes. However the author was around to assist the participants if they have any difficulty or question to ask.

\section{Results and Discussion}

\subsection{Result}

As explained in the previous section, evaluation sessions were carried out to find out users satisfaction on the system. Table 1 shows the demographic statistics of the participants'. There were no relevant demographic differences between the participants in each of groups (Note 1).

Based on Figure 9 (Note 1), there are three (3) main things that the author wants to know about the Notification System from the respondents, efficiency, usability and functionality:

- Efficiency: $80 \%$ of the students and $70 \%$ of the System Analysts think that the system is efficient and it is good enough. This might be because of the time taken to test all the function was quite fast, since the system was designed properly and the hardware was quite high performance.

- Usability: $70 \%$ of the students and $90 \%$ of the System Analysts think that the system is usable and easy to use. Some of the students believe that the system is not very easy to use because due to their less exposure to system like this.

- Functionality: $90 \%$ of the students and $70 \%$ of the System Analysts think that the system runs as it should and has all the necessary functions. The System Analysts were not very satisfy with the functionality might be because of they are familiar with this kind of system and they expected more from this system.

Among the significant suggestions given by the participants are:

- $\quad$ The layout design is too crowded. They think that it should be more relax and spacious.

- $\quad$ The page is too lengthy, that they have to scroll down every time they want to read article at the bottom.

They think that the height of the page should be fixed and fit to the browser height.

- The system should provide chat function so that researchers can communicate among them while browsing the system.

Based on the findings, there are a few things that can be concluded:

- $\quad$ Most of the participants satisfy with the system usability, functionality and efficiency. 
- $\quad$ Most of the participants believe that this system should be used by the Bioinformatics CoP as a tool for them to communicate and cooperate in research as it shown in table 1 (Note 1).

- The notification functionality in this system helps the users in using the system efficiently and doing their research effectively as it shown in table 1 (Note 1).

- $\quad$ Most of the participants agree that this system has all it takes to be a good system as they expected as it shown in table 1 (Note 1).

\subsection{Discussion}

On the whole, Notification System for Bioinformatics CoP has fulfils its objective and successfully completed according to the research scope. This notification system obviously helps the user in selecting the right article to read and also remind them on their schedule. These advantages can encourage the Bioinformatics CoP to take more active participation in the community's portal, thus can enhance research in Bioinformatics.

The agent technology certainly helps to make the notification functionality in this system a reality. The technology provides the system the ability to work, 'think' and act on its own to achieve its goal. This technology is essential in notification research area, so more study need to be done to strengthen it, and thus benefits the society at the end of the day.

Based on the evaluation done by the 20 participants, we can see that the system is good enough in term of functional and non functional. All the functions that were planned in the early chapters managed to be accomplished and worked as it should. While the non functional criteria such as the system pleasantness of layout design and easiness of using the system did also catch the testers' attention. Although there are some enhancement comments to the system, we believe that they are our opportunities to improve the system in the future.

\section{Conclusion}

Knowledge Management System (KMS) has been widely implemented by organization in order to keep the most valuable asset, which is the knowledge. The implementation of KMS in the bioinformatics community is also important due to its huge area and promising potential. However implementing KMS in Bioinformatics community is not as easy as it seems because a few factors need to be considered to make sure it is a success not the other way round.

Among the factors is to make sure that the KMS is really assisting the users in this research community and one of the way is through notification. Notification can exists in many forms and manners, but in this project we focused on notifying the users in selecting the most appropriate articles or knowledge to be read and notifying them on their important events. By assisting the users in these manners, we can attract them to make this system a place where they can communicate and work together as a cyber research community.

As a result it is crucial to implement the Notification System in the Bioinformatics CoP to boost up research in Bioinformatics and promote UPM as a leader in Bioinformatics research. Based on the survey conducted on the participants which we had a very positive feed backs, it is believed that the system is good and worth enough to be implemented in the Bioinformatics CoP.

For future works, it is a good idea if the current system could be enhanced to notify users on other Bioinformatics resources. Currently when a user is reading an article on something, the system will list down all other articles available in the system that are correlated to that particular article. It is a good thing if in the future the system could also suggest other articles, links, videos, white papers and etc from other resources. Besides benefiting the users with great knowledge, it could increase the system's knowledge databanks with unlimited source of knowledge.

\section{References}

Abdulla R. (2008). "Knowledge Management System in a Collaborative Environment", Penerbit Universiti Putra Malaysia, Serdang, 344-355.

Bioplanet. (2009). The Bioinformatics Homepage, http://www.bioplanet.com/Accessed on 23/03/2009.

Bioinformatics Organization. (2009). http://www.bioinformatics.org/, Accessed on 23/03/2009.

Bruegge B. \& Dutoit A. H. (2004). "Object Oriented Software Engineering (Using UML, Patterns and Java)" Second Edition, Pearson Prentice Hall,. Chapter 4.

Carzaniga A., Rosenblum D. S., Wolf A. L. (2001). "Design and Evaluation of a Wide-Area Event Notification Service", ACM Transactions on Computer Systems, Vol. 19, No. 3, Pages 332-383. 
CGB Bioinformatics Portal-The Centre For Genomic and Bioinformatics, http://bioportal.cgb.indiana.edu/, Copyright 2000-2010: The Center for Genomics and Bioinformatics, and The Trustees of Indiana University, Accessed on 08th April 2009.

Heylighen F. (2002). "Complexity and Information Overload in Society: why increasing efficiency leads to decreasing control”, The Information Society.

InfoRouter-Knowledge_Management, http://www.inforouter.com/Knowledge_Management.asp, Accessed on 23th Mac 2009.

Jacques C. (2004). "Bioinformatics-An Introduction for Computer Scientists", ACM Computing Surveys, Vol. 36, No. 2, pp. 122-158.

Morrow K. J. (2008). “Can In Silico Modeling Speed Development?”, Genetic Engineering and Biotechnology News, Vol. 28, No. 13.

Nwana H. S. (1996). "Software Agents: An Overview”, Knowledge Engineering Review, Vol. 11, No 3, pp.1-40, Cambridge University Press.

Pressman R.S. (2001). "Software Engineering - A Practitioner's Approach", 5th Ed., McGraw-Hill. Chapter 3.

Rodríguez O. M., Vizcaino A. (2004). "Using a Multi-Agent Architecture to Manage Knowledge in the Software Maintenance Process", Lecture Notes in Artificial Intelligence 3213: 1181-1187.

Russell S., \& Norvig P,A. (1995). “ Modern Approach by Prentice” Hall, Inc

Wageningen Bioinformatics Webportal, Last Modified July 22, 2009 by JL, http://www.bioinformatics.nl/, , Accessed on 03th April 2009.

Table 1. Demographic characteristic

\begin{tabular}{|c|c|c|c|}
\hline \multicolumn{4}{|c|}{ Demographic characteristics of the participants } \\
\hline \multirow[t]{2}{*}{ Gender } & Male & Female & \\
\hline & $60 \%$ & $40 \%$ & \\
\hline \multirow[t]{2}{*}{ Age } & $<25$ years & 26-30 years & $>30$ years \\
\hline & $20 \%$ & $25 \%$ & $55 \%$ \\
\hline \multirow[t]{2}{*}{ Occupation } & Master Student & System Analyst & \\
\hline & $50 \%$ & $50 \%$ & \\
\hline \multirow[t]{2}{*}{ Experience in using computer } & $<1$ year & $1-5$ years & $>5$ years \\
\hline & $0 \%$ & $5 \%$ & $95 \%$ \\
\hline
\end{tabular}

Table 1 above summarized the basic characteristics of the initial sample as well as those of the participants'. There were no relevant demographic differences between the participants in each of groups. As can be noted from the table above, the sample is rather skewed towards males in the age group between 25 and 30 as well as the female too and of their Occupation its equivalent for both $(50 \%=50 \%)$. About their experience in using computer both of them got $95 \%$ over 5 years experience. 


\section{Notes}

Note 1

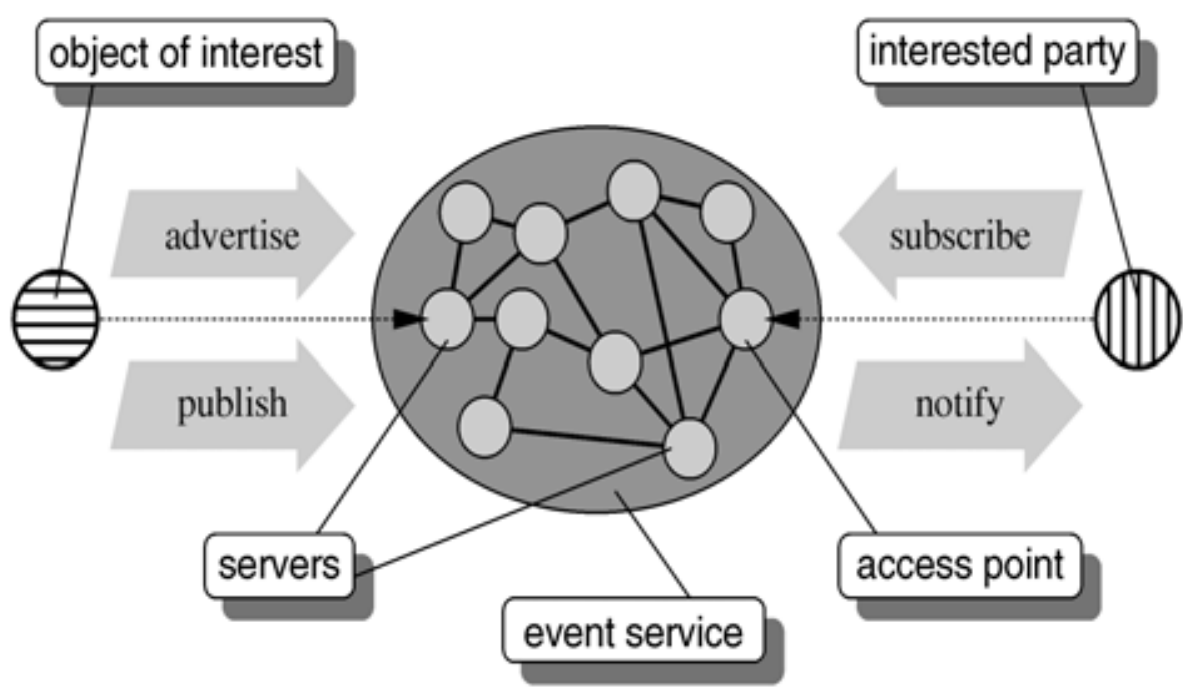

Figure 1. Distributed event notification service

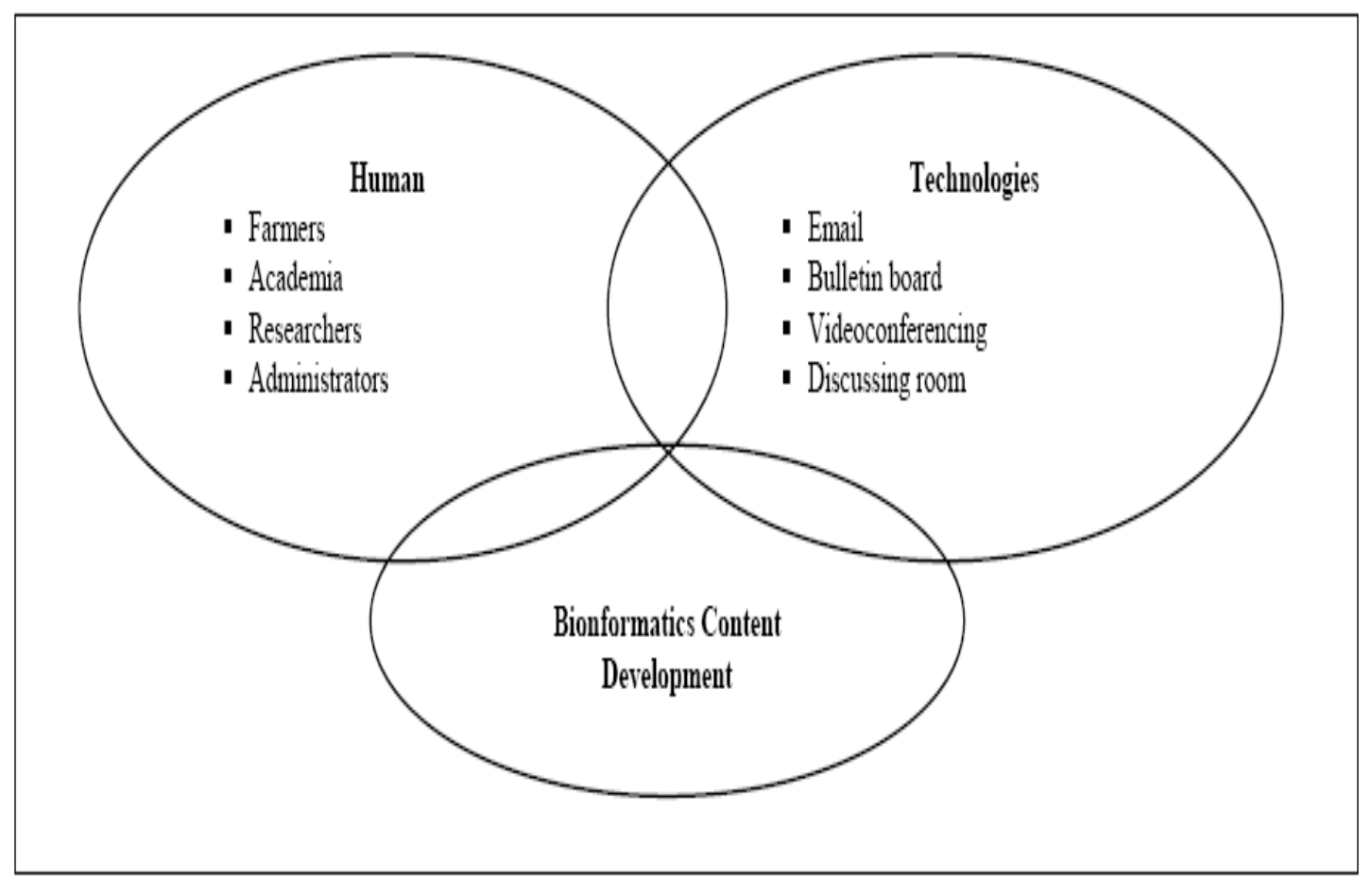

Figure 2. The System Components of Knowledge Management Framework for Bioinformatics Institutions 


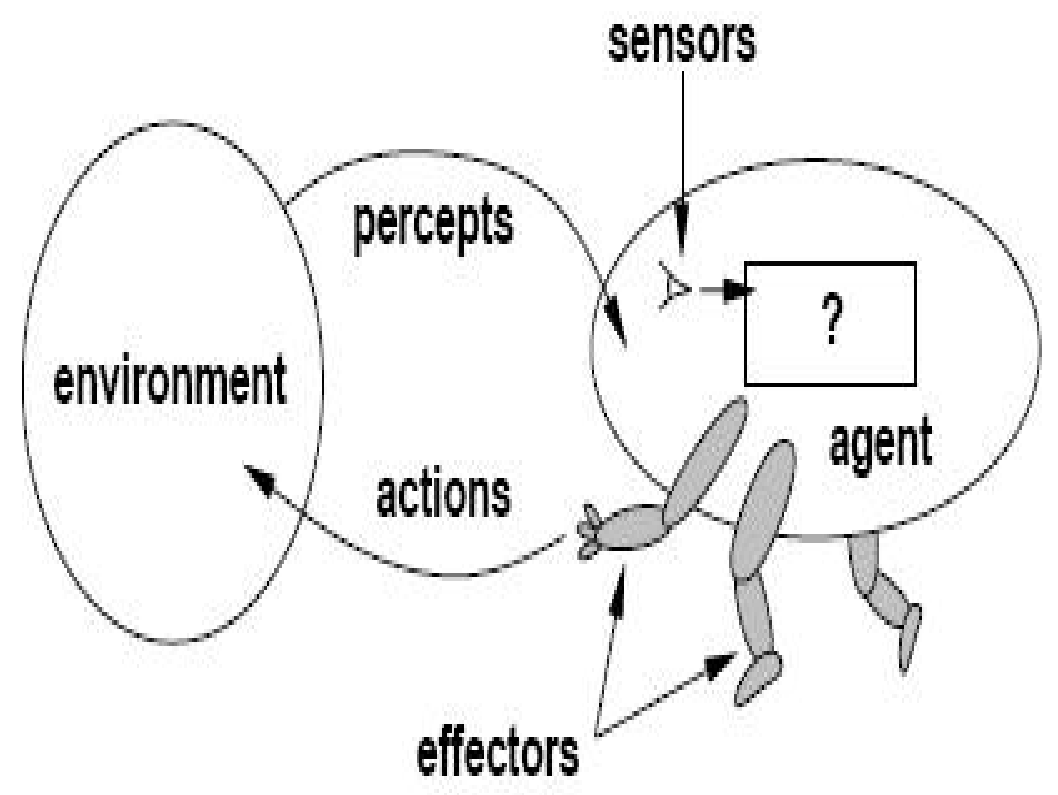

Figure 3. Interaction between Agent and Environment

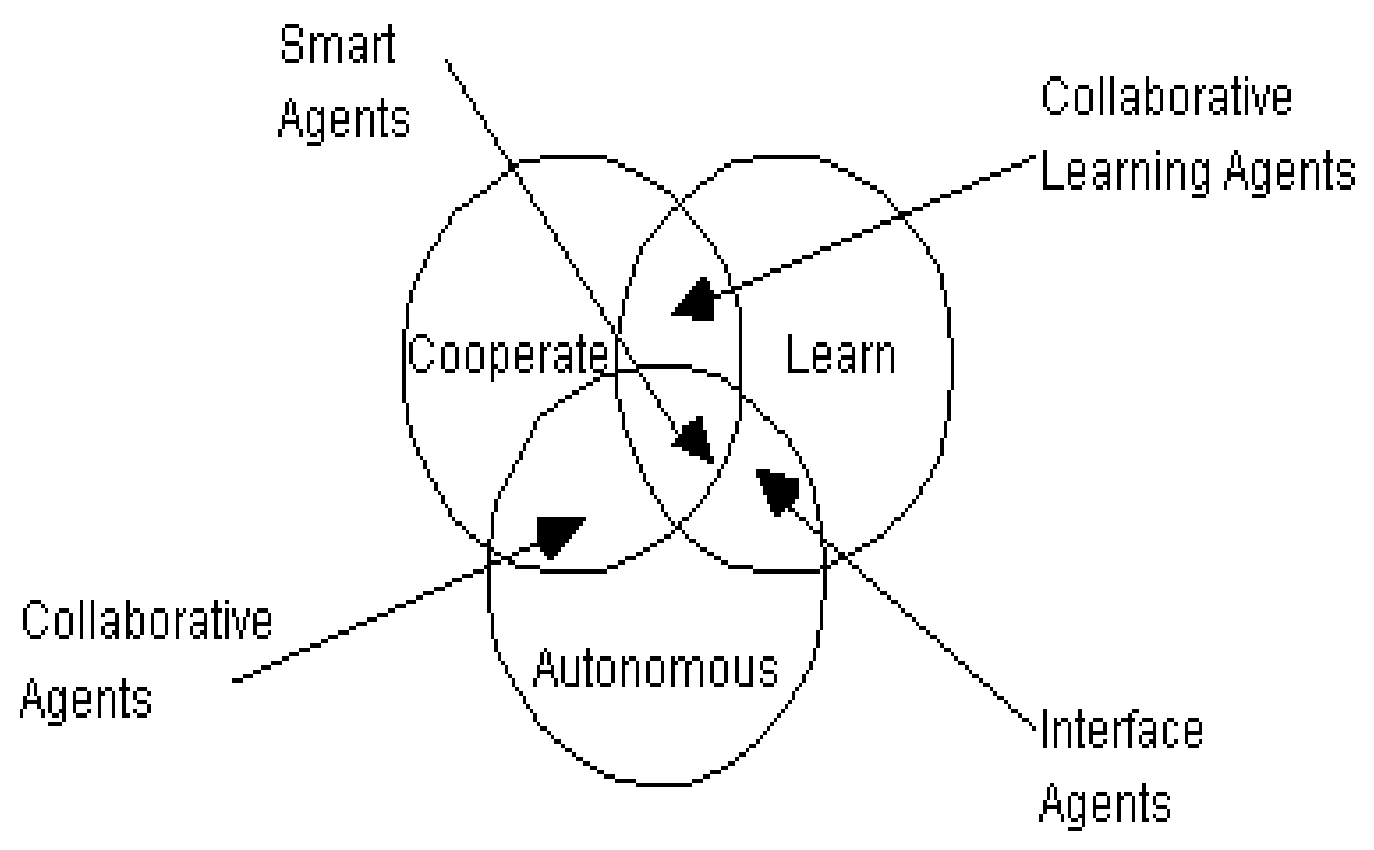

Figure 4. Agent Topology 


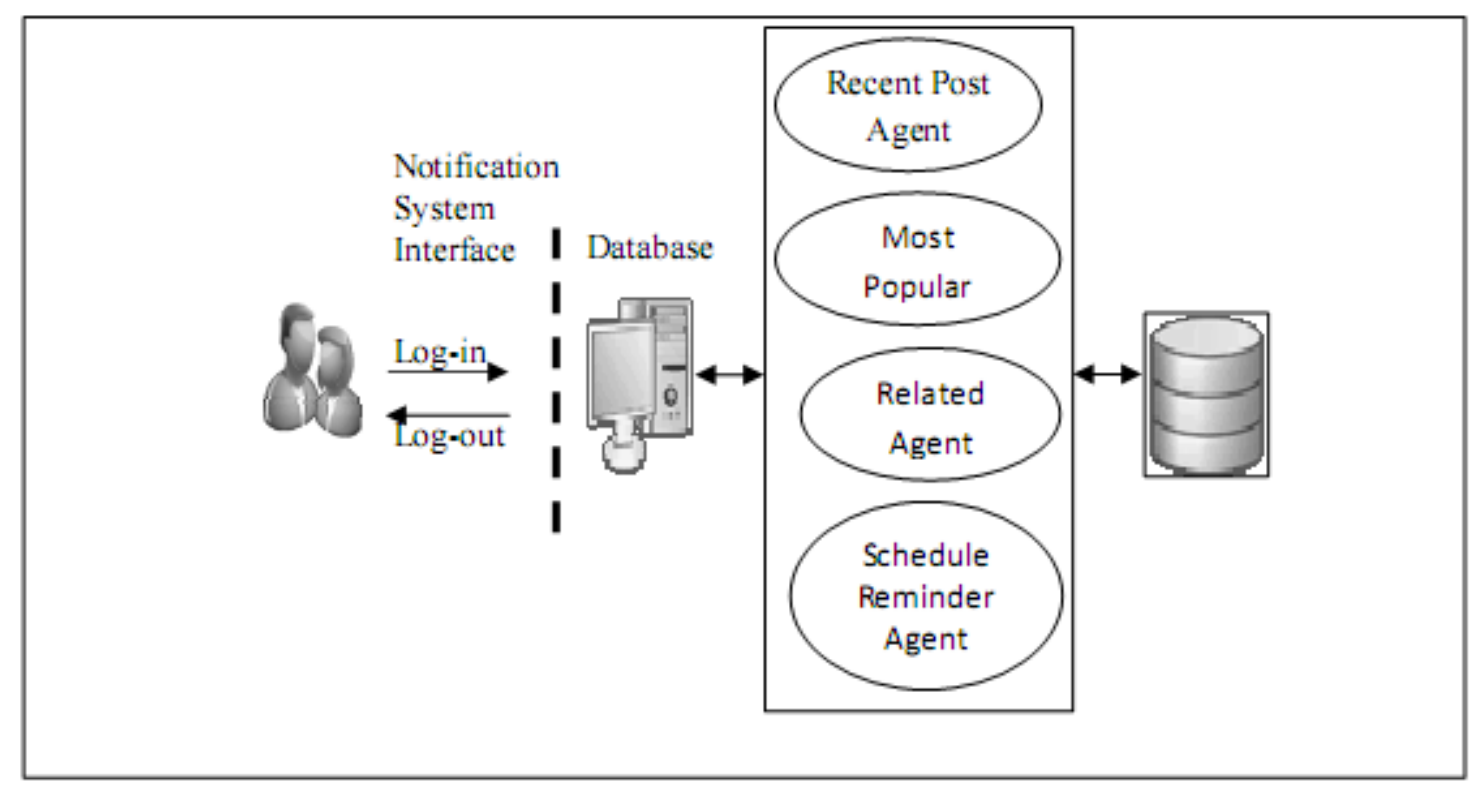

Figure 5. System Architecture

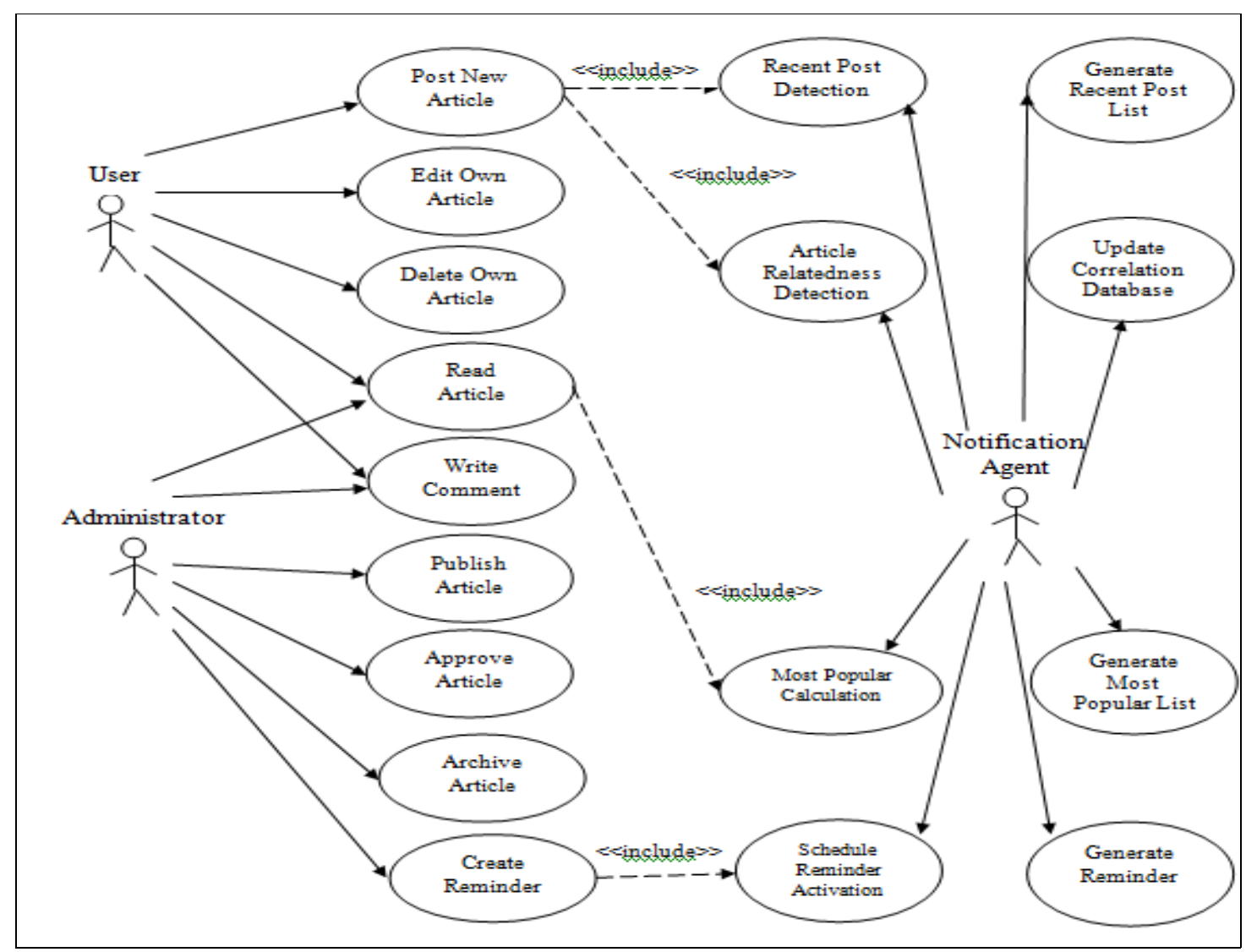

Figure 6. Notification System Use Case Diagram 


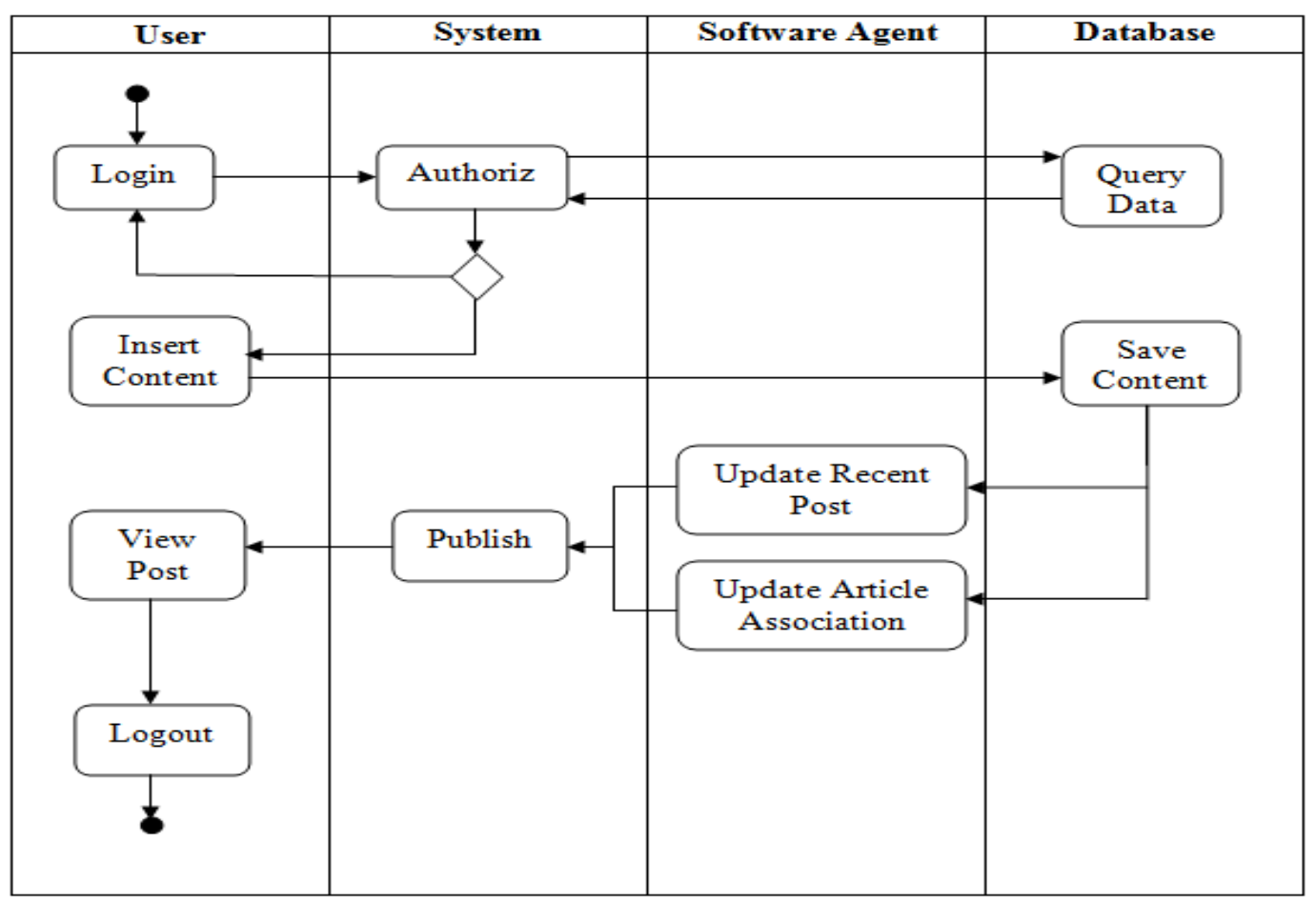

Figure 7. Activity Diagram of Posting New Article

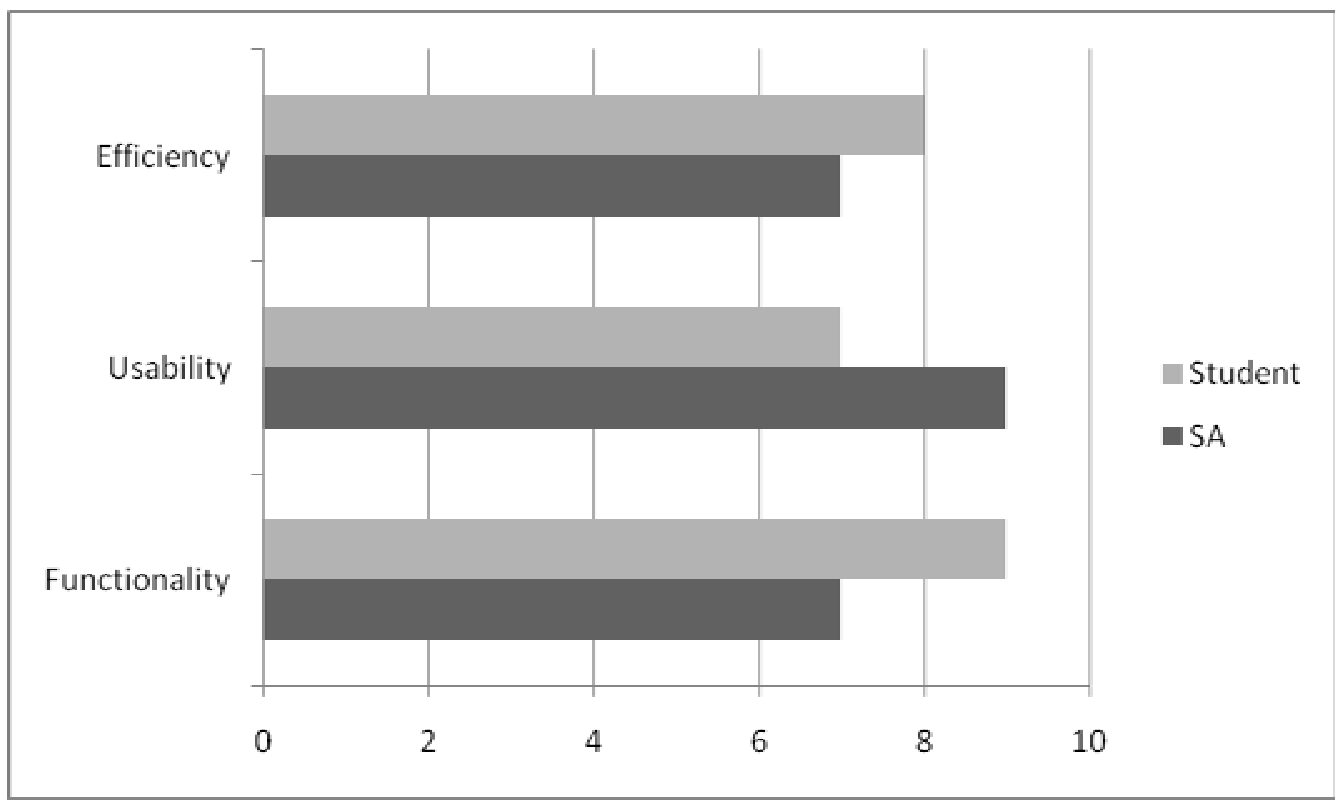

Figure 9. Evaluation result 
Note 2

Appendices

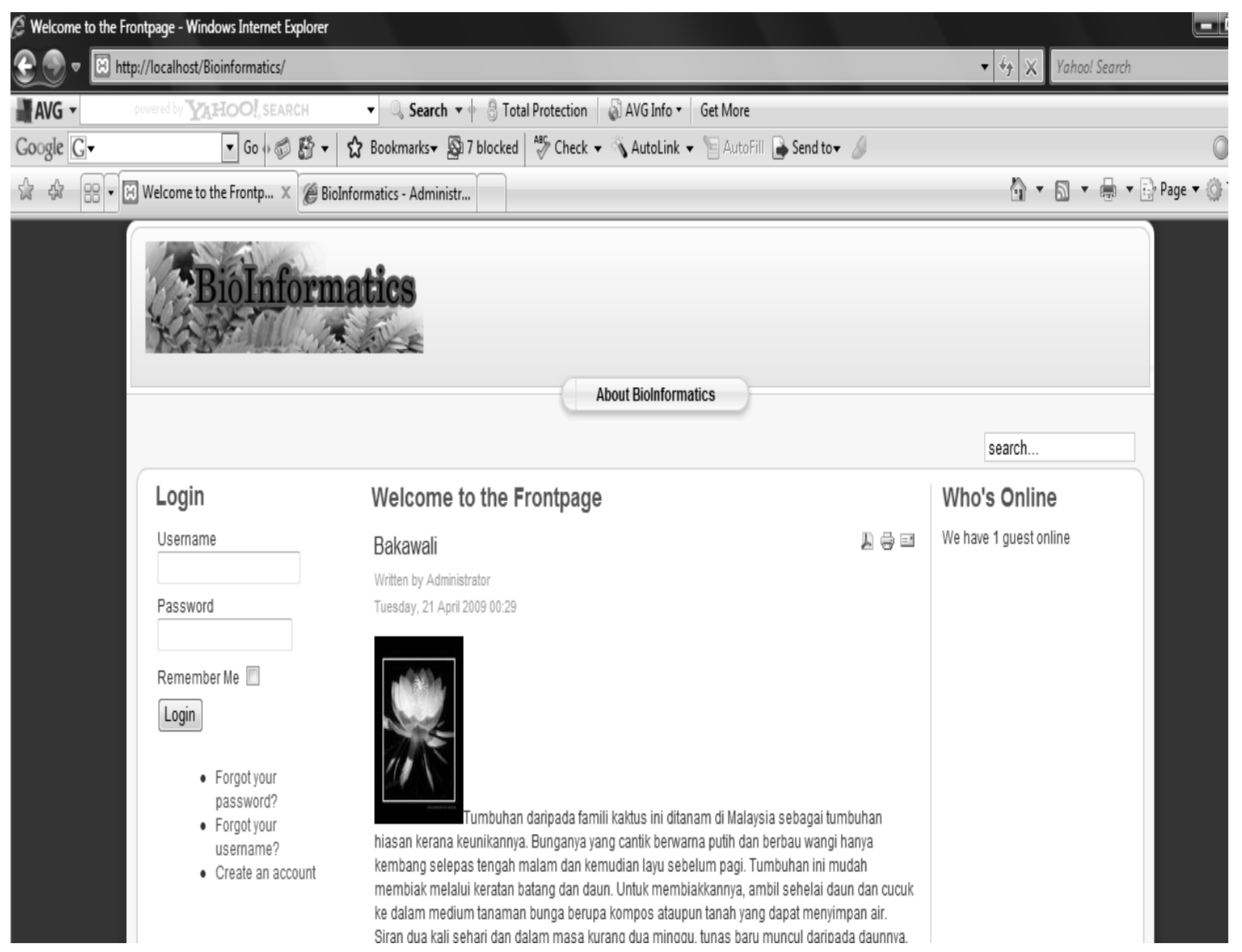

Figure A. Login Page 


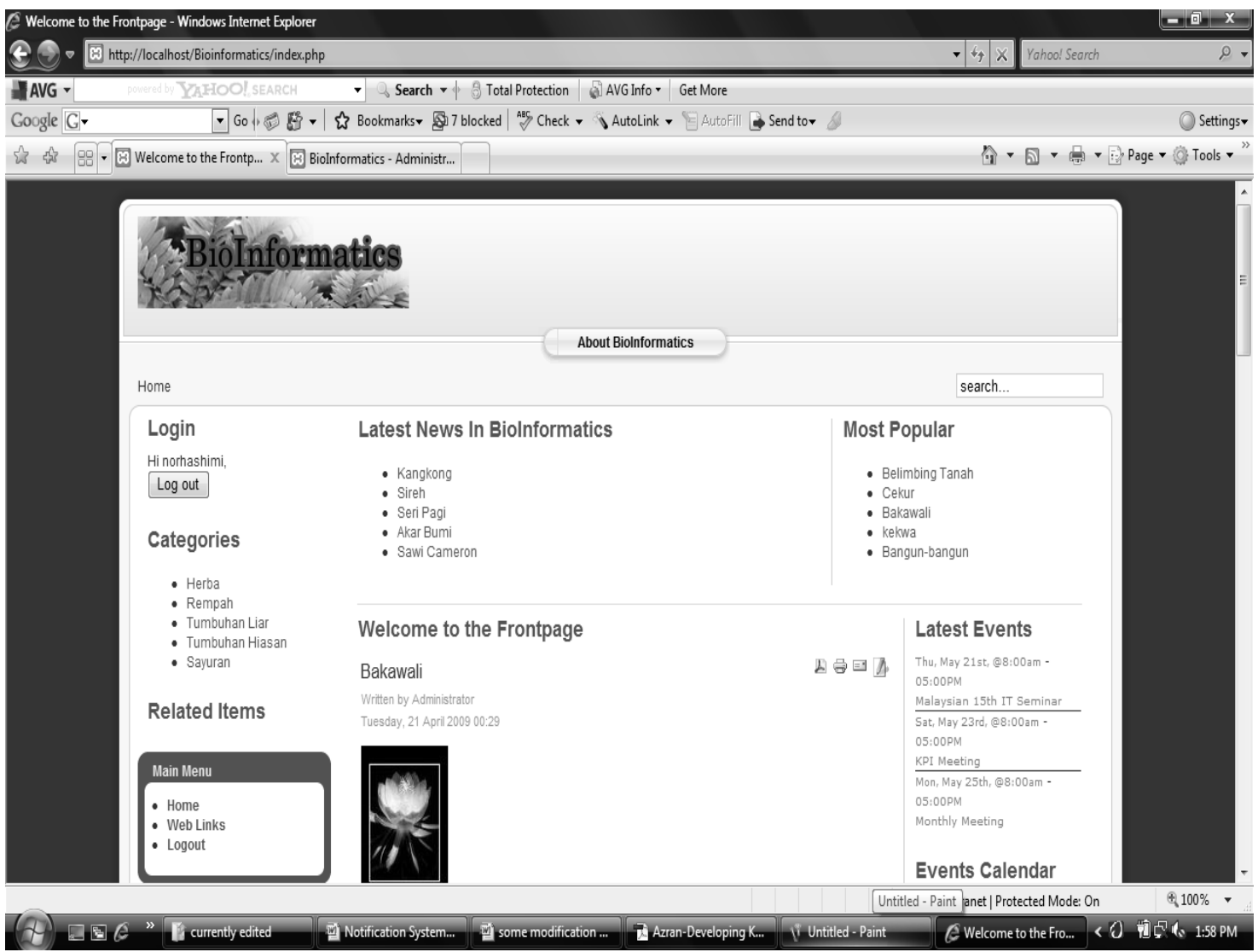

Figure B. Main Page (Top)

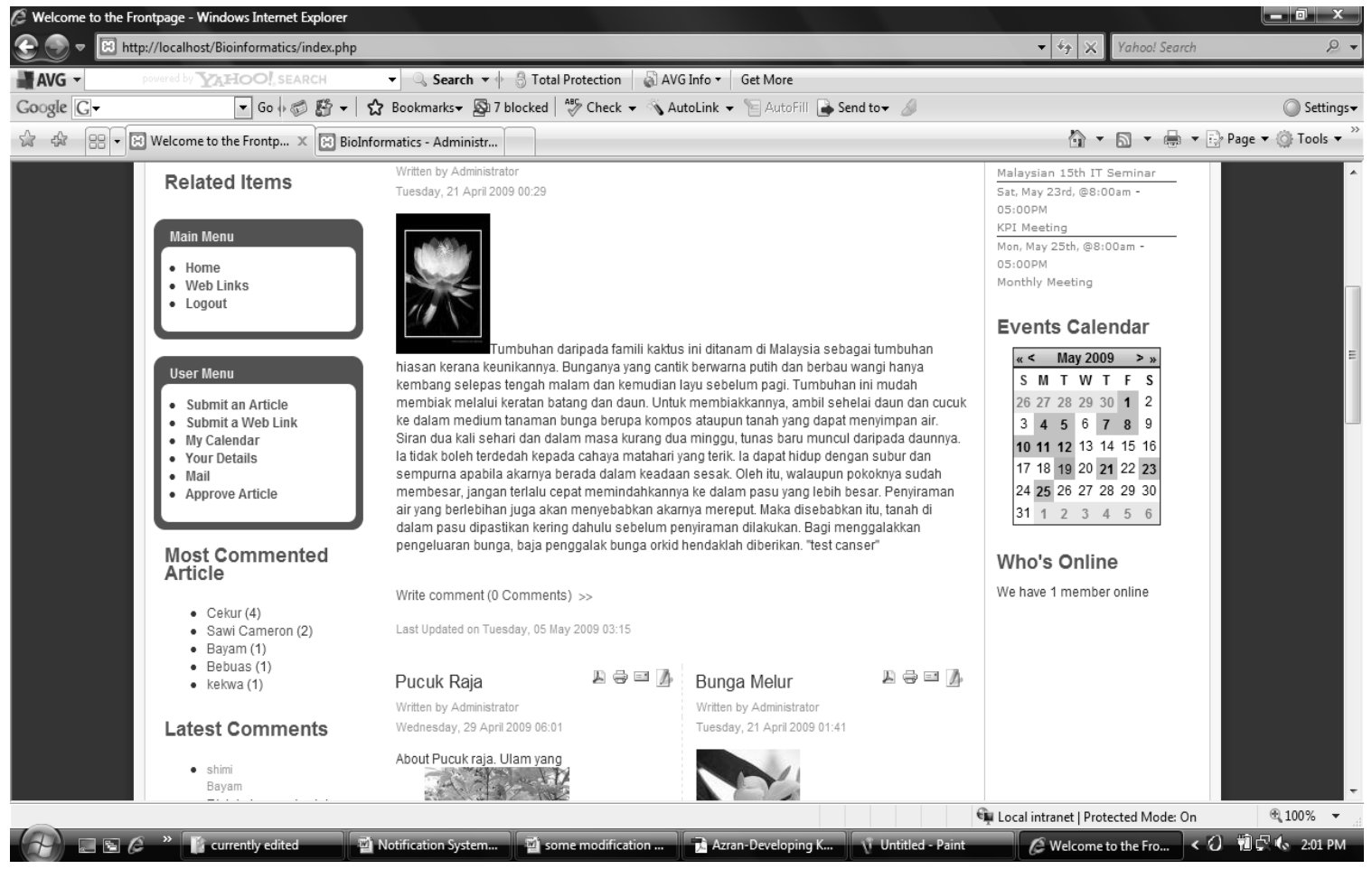

Figure C. Main Page (Bottom) 


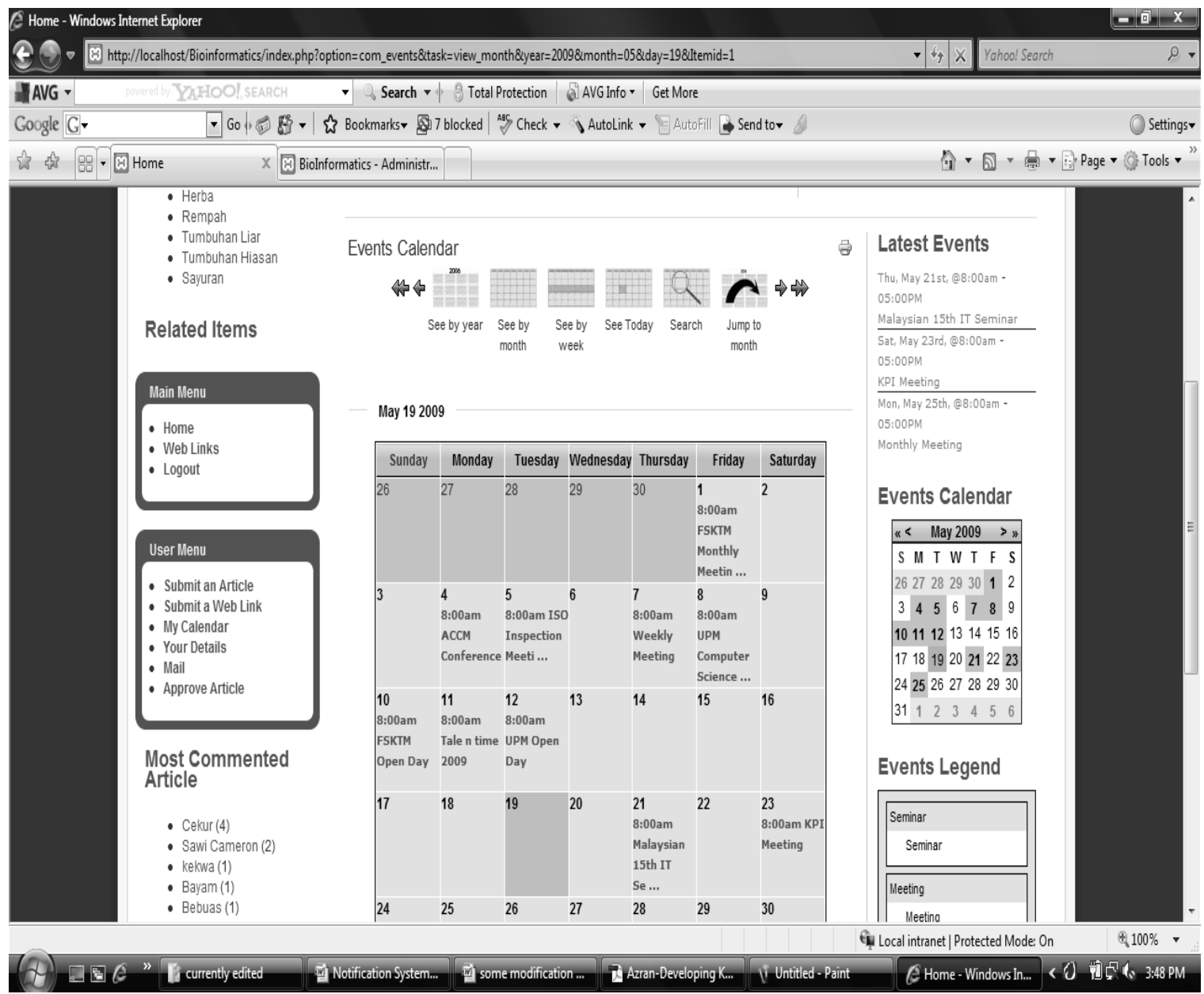

Figure D. Community Events 